\title{
Orbital Metastasis from Breast Cancer Without Significant Changes in CT Scan and MRI
}

\author{
Payam Azadeh, ${ }^{1}$ Babak Hassanzadeh Rad, ${ }^{2}$ and Ali Yaghobi Joybari1 ${ }^{1,}$ \\ ${ }^{1}$ Department of Radiation Oncology, Shahid Beheshti University of Medical Sciences, Tehran, Iran \\ ${ }^{2}$ Veterinary Medicine Department, Karaj Branch, Islamic Azad University, Karaj, Iran \\ "Corresponding author: Ali Yaghobi Joybari, Department of Radiation Oncology, Shahid Beheshti University of Medical Sciences, Tehran, Iran. Tel/Fax: +98-2122859943, E-mail: \\ dryaghobii@yahoo.com
}

Received 2014 May 24; Revised 2014 July 23; Accepted 2014 August 09.

Keywords: Breast Cancer, Orbital Metastasis, CT/MRI, OCT

\section{Dear Editor,}

Orbital metastasis infrequently occurs in breast cancer (1); however breast cancer is the most common cause of orbital metastasis (2-5), followed by lung, prostate, gastrointestinal tract, and skin (melanoma) cancers $(6,7)$.

Definite diagnosis of an orbital lesion includes an orbital biopsy (either FNA or open biopsy). However, in patients with known metastatic cancer, CT scan or MRI of the orbits can frequently show the presence of a mass, which often involves the orbital fat or extraocular muscles $(4,5)$. On the other hand, these techniques may not completely reflect the metastases. Optical coherence tomography (OCT) is another method. It is a non-contact, non-invasive and high-resolution technique for imaging. There are two types of OCT, including time-domain (such as Stratus OCT3) and frequency-domain (same as Spectralis HRA+OCT). Frequency-domain is an advanced technique in imaging speed and diagnostic sensitivity (8). The Spectralis HRA+OCT produces a unique combination of retinal angiography and optical coherence tomography. Here, we report a case of orbital metastasis in a woman with previously diagnosed metastatic breast cancer presenting with visual disturbance of the right eye which was detected by optical coherence tomography.

A 53-year-old woman presented with a 2-month history of diplopia and decreased vision in her right eye. Her past medical history included stage 2 , hormone-receptorpositive and HER2-negative left breast carcinoma approximately 3 years prior to her presentation. She underwent modified radical mastectomy and axillary lymph node dissection. She subsequently received chemoradiation followed by hormonal therapy. Two years later, she presented with dyspepsia and abdominal pain. An abdominopelvic computed tomography (CT) scan showed ascites, peritoneal seeding, mucosal thickening, and partial obstruction in the proximal and distal part of the stomach, which was confirmed by endoscopy. In addition, ascites analysis was positive for malignancy. Chemotherapy was instituted which led to complete response. Then she traveled to England and her treatment was continued by hormone therapy. Six months later, she returned with complaints of abdominal pain, headache and unilateral visual disturbance, including diplopia and decreased visual acuity in the right eye. A repeated abdominopelvic CT scan revealed ascites and peritoneal seeding. Neurological examination failed to reveal any focal neurological deficit. Ophthalmologic examination revealed that the patient's visual acuity was approximately no light perception (NLP) in the right eye. CT scan with and without contrast and magnetic resonance imaging (MRI) of the orbits and head were performed, which indicated no significant change (Figure 1). The type of MRI machine was Philips 1Tesla intera and the MRI technique was axial proton density (PD), axial T1W before injection of contrast, axial and sagittal T1W non fat suppress after injection of contrast media. Generally, on MRI, metastatic lesions to the orbit present hypointense to fat on T1W and hyperintense to fat on T2W, which was not observed in our case. Furthermore, nuclear scan was negative for metastasis to the globe.

However OCT (Spectralis HRA+OCT) findings were most compatible with metastatic disease in vitreous and head of the optic nerve. OCT of orbital metastasis indicates a dome shaped elevation of the neurosensory retina and retinal pigment epithelium (RPE) with adjacent subretinal fluid. It could also be associated with retinal edema, intraretinal cysts, and thickening and detachment of the RPE. About $14.2 \%$ of cases have shown highly reflective points within neurosensory detachment. These points may correspond to retinal compromise by cancer cells or macrophages containing lipofuscin and melanin granules $(9,10)$. A combined chemotherapy treatment with paclitaxel and carboplatin for a total of 11 cycles was started. Eye 

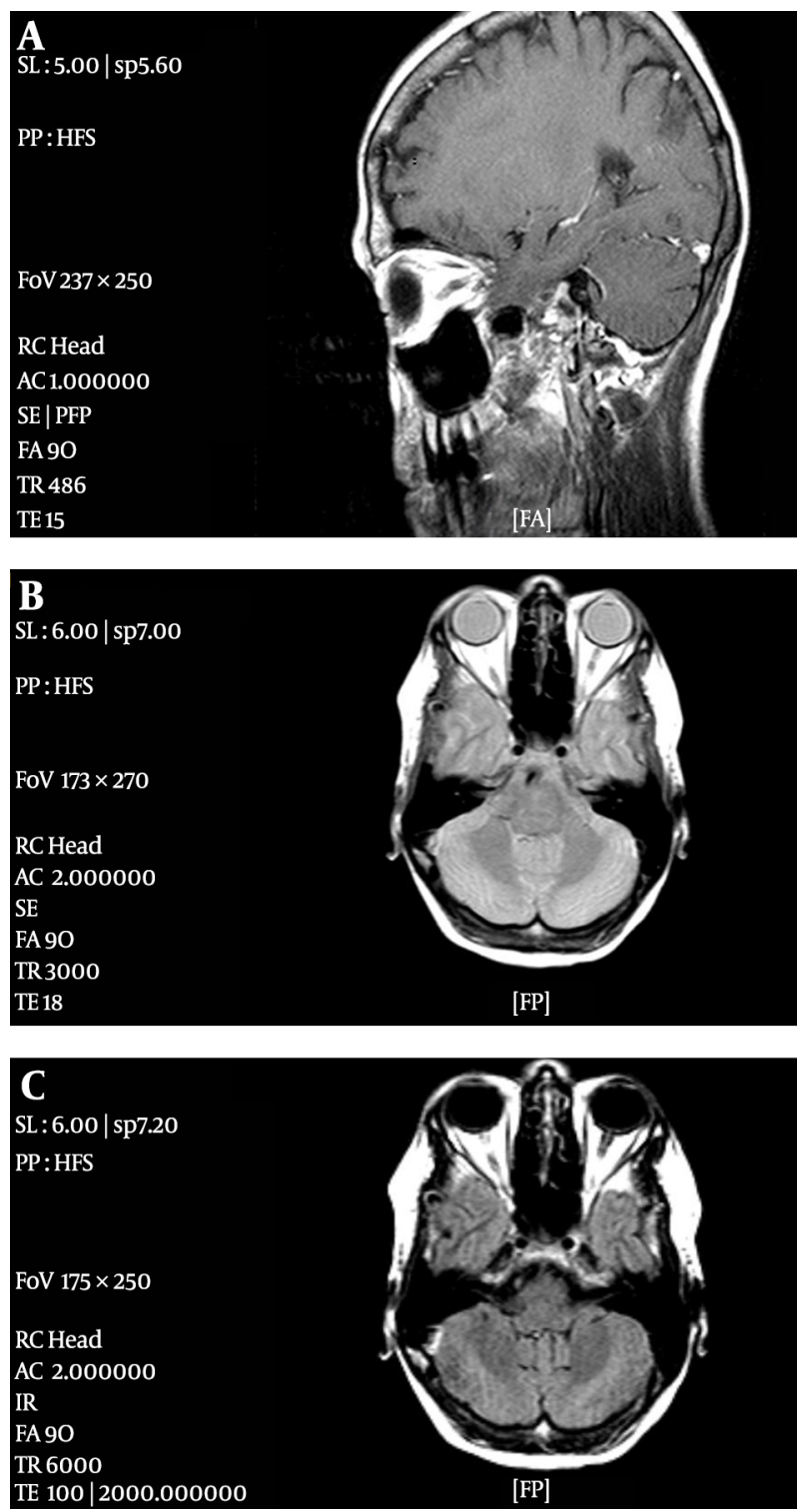

Figure 1. Head and orbit MRI did not show any significant change

symptoms resolved almost completely during the following weeks. At present, the patient' visual acuity is 10/10 in the right eye.

We have assessed and followed up the patient by clinical and ophthalmological examinations and imaging techniques including CT scan (with or without contrast) of the abdominopelvis region, MRI (with or without contrast) of the cervical spine and orbit, and mammography, which did not detect any significant problems.

One of our limitations was using 1Tesla MRI machine and the above mentioned protocols. Asymmetry of the posterior wall of the globes or any other changes may be visible with 1.5 or 3 Tesla MRI and complete protocols by special orbital cuts and sequences.

In summary, increased vigilance on behalf of the oncologic and ophthalmologic communities is required when they observe symptoms and signs compatible with ocular disease in patients with an established diagnosis of breast cancer. In addition, if CT scan or MRI of the orbits did not show presence of a mass, use of OCT or biopsy should be considered.

\section{Footnotes}

Authors' Contributions: Study concept: Payam Azadeh and Ali Yaghobi Joybari; data gathering: Ali Yaghobi Joybari; drafting of the manuscript: Babak Hassanzadeh Rad.

Financial Disclosure: We did not have any relevant financial interests or financial conflicts within the past 5 years and for the foreseeable future.

Funding/Support: We received no financial support for the research, authorship, and or publication of this article.

\section{References}

1. Tamura M, Tada T, Tsuji H, Tamura M, Yoshimoto M, Takahashi K, et al Clinical study on the metastasis to the eyes from breast cancer. Breast Cancer. 2004;11(1):65-8. [PubMed: 14763463].

2. Shields JA, Shields CL, Brotman HK, Carvalho C, Perez N, Eagle RJ. Cancer metastatic to the orbit: the 2000 Robert M. Curts Lecture. Ophthal Plast Reconstr Surg. 2001;17(5):346-54. [PubMed: 11642491].

3. Valenzuela AA, Archibald CW, Fleming B, Ong L, O'Donnell B, Crompton JJ, et al. Orbital metastasis: clinical features, management and outcome. Orbit. 2009;28(2-3):153-9. [PubMed: 19839900].

4. Freedman MI. Metastatic Tumors to the Eye and Orbit. Arch Ophthalm. 1987;105(9):1215. doi:10.1001/archopht.1987.01060090073031.

5. Shields JA, Shields CL, Scartozzi R. Survey of 1264 patients with orbital tumors and simulating lesions: The 2002 Montgomery Lecture, part 1. Ophthalmology. 2004;111(5):997-1008. doi: 10.1016/j.ophtha.2003.01.002. [PubMed: 15121380].

6. Ahmad SM, Esmaeli B. Metastatic tumors of the orbit and ocular adnexa. Curr Opin Ophthalmol. 2007;18(5):405-13. doi: 10.1097/ICU.0b013e3282c5077c. [PubMed:17700235].

7. Holland D, Maune S, Kovacs G, Behrendt S. Metastatic tumors of the orbit: a retrospective study. Orbit. 2003;22(1):15-24. [PubMed:12759863].

8. Huntjens B. Tracking laser tomography. Optician. 2009:12-5.

9. Natesh S, Chin KJ, Finger PT. Choroidal metastases fundus autofluorescence imaging: correlation to clinical, OCT, and fluorescein angiographic findings. Ophthalmic Surg Lasers Imaging. 2010;41(4):406-12. doi: 10.3928/15428877-20100426-03. [PubMed: 20438045].

10. Arevalo JF, Fernandez CF, Garcia RA. Optical coherence tomog raphy characteristics of choroidal metastasis. Ophthalmology. 2005;112(9):1612-9. doi: 10.1016/j.ophtha.2005.03.030. [PubMed: 16039718]. 